\title{
UN PAESE DA SCOPRIRE, UNA TERRA DA AMARE. PAESAGGI EDUCATIVI E FORMAZIONE DELL'IDENTITÀ NAZIONALE NELLA PRIMA METÀ DEL NOVECENTO
}

Por Fabio TARghetta. Milano: Franco Angeli, 2020, 203 páginas. ISBN: 978-8891799364

Gli osservatori che dall'estero guardano alla storia dell'Italia sono spesso sorpresi dalla quantità di studi e ricerche che hanno approfondito lo sforzo profuso dalle élites liberali per «fare gli italiani». Ma la ragione è presto spiegata: l'Italia ha alle spalle una storia frammentata in tante storie regionali e municipali - non unitaria come è invece accaduto per altri Paesi come, ad esempio, la Francia - e una prospettiva nazionale coltivata nei secoli soprattutto come storia letteraria intorno a cui in età romantica si andò costituendo l'idea di nazione. E, dunque, appena fatta l'Italia, fu necessario mettere mano alla formazione della coscienza dei cittadini.

Quando l'Italia nel 1861 si costituì come Stato indipendente la consapevolezza di essere una nazione apparteneva infatti a una ristretta élites di intellettuali, di patrioti liberali, di ricchi borghesi. La grande massa della popolazione era stata estranea al processo che aveva portato all'unificazione ed era estranea al nuovo Stato. Forte era il radicamento nelle varie e diverse culture sparse sul territorio italiano. Soltanto una ridotta parte dei nuovi italiani parlava la lingua nazionale, in grande maggioranza la comunicazione avveniva attraverso le parlate dialettali. Pesi e misure restarono a lungo quelli tradizionali e ancora a fine Ottocento/inizio Novecento molte transazioni avvenivano sulla base delle consuetudini locali.

Se si tiene presente questo stato di cose si comprende come la conquista dell'identità nazionale collettiva fu un processo complesso, lungo, 
irto di ostacoli anche in conseguenza dei difficili rapporti tra lo Stato e la Chiesa. Per molti decenni, fin quasi alla prima guerra mondiale, i cattolici non si sentirono «italiani» in senso patriottico, ma «italiani» fedeli prima di tutto al Papa. Il ceto dirigente non lesinò gli sforzi per nazionalizzare le masse popolari e mise di conseguenza in campo molteplici strategie finalizzate a tale scopo, dalla scuola elementare obbligatoria al servizio militare, dalle cerimonie pubbliche accompagnate da grande sfarzo alla costruzione di monumenti celebrativi dei personaggi più in vista del Risorgimento nazionale fino alla costruzione del Pantheon dei cosiddetti «padri della Patria» (Cavour, Garibaldi, Mazzini, Vittorio Emanuele II).

Sono queste le ragioni che spiegano perché il tema della formazione degli italiani ha occupato largo spazio nelle ricerche storiografiche del passato. Il libro di Fabio Targhetta rientra autorevolmente e precisamente in questo importante filone di studi. L'originalità del suo lavoro consiste nel tema considerato e nel modo con cui vengono indagati la rappresentazione del paesaggio e il suo impiego a scopi educativi. L'autore li sintetizza nell'espressione "paesaggi educativi», ovvero l'insieme assai multiforme di ambienti e contesti oggetto di pratiche didattiche cariche di finalità identitarie, forme di mediazione, in altre parole, che favoriscono in scolari e studenti «l'attribuzione eterodiretta di significati al territorio in direzione del rafforzamento del senso di appartenenza».

Scopo della ricerca di Targhetta è infatti quello di approfondire tempi, modalità e caratteristiche mediante le quali il paesaggio - nelle sue varie modulazioni: raccontato, raffigurato, sperimentato praticamente (questi sono i temi analizzati in altrettanti capitoli del libro) - abbia contribuito alla formazione della coscienza nazionale, ricorrendo a una grande varietà di stimoli - da quelli più culturali ad altri più sentimentali -, a partire dal patriottismo veicolato dalla ricorrente affermazione che «L'Italia è il paese più bello del mondo».

La narrazione si svolge appoggiandosi a una notevole varietà di fonti in chiave multi e interdisciplinare che spaziano da quelle più specificamente scolastiche (programmi di insegnamento, libri di testo, quaderni, carte geografiche, materiale visivo, etc.) a fonti e ricerche condotte in ambito linguistico, geografico, semiotico, sociologico con uno sguardo anche attento agli innovativi contributi apparsi negli ultimi decenni su questi temi a livello internazionale. 
Scorrono così nelle pagine del libro, per citare qualche esempio, l'interesse rivolto all'insegnamento della geografia spesso intrecciato con quello storico, alla descrizione delle grandi città nei libri di testo, all'uso ideologico della nozione di confine, alle riproduzioni fotografiche e filmiche degli scorci paesaggistici e dei monumenti più importanti dell'Italia, alla "pedagogia del turismo» (escursioni e viaggi d'istruzione) e al ruolo del Touring club, alla funzione dell'architettura funeraria in specie dopo la prima guerra mondiale.

Tra i tanti spunti degni di segnalazione contenuti nei primi capitoli del libro vorrei indicare come meritevoli di particolare attenzione le riflessioni sul paesaggio ai confini del Regno perché proprio l'impiego ideologico del confine favorisce l'approccio nazionalistico volto a superare lo scarto tra lo Stato e la Nazione e la conseguente martellante rivendicazione di territori ritenuti geograficamente italiani («terre italiane che non fanno parte del Regno d'Italia»: ad esempio Corsica, Trentino e Sud Tirolo, Canton Ticino, Istria, fino addirittura l'isola di Malta). Siamo in presenza di qualcosa di più di una sana educazione patriottica con la messa a punto delle condizioni per la diffusione di una capillare mentalità nazionalistica alla base, tra altre motivazioni, dell'affermazione del fascismo che fece delle rivendicazioni territoriali uno dei suoi motivi portanti.

Il paesaggio è infatti spesso occasione oscillante, come annota giustamente l'autore, tra la coltivazione dei valori patriottici necessari per interiorizzare la nozione di nazione e l'esaltazione nazionalistica che assume caratteri particolarmente evidenti tra le due guerre segnate dal ventennio fascista.

Nella seconda parte del libro Targhetta si sofferma sui tre paesaggi propri dell'Italia - l'ambiente urbano, il mondo contadino e la realtà della montagna - per coglierne le specificità e i valori di cui sono portatori, le trasformazioni economiche e sociali che nel tempo li hanno modificati e le ragioni che ne sostengono le caratteristiche di «paesaggi aggregatori di emozioni capaci di suscitare, se opportunamente stimolati, sentimenti di attaccamento e di orgoglio».

Per quanto riguarda il paesaggio cittadino gli interventi che ne esaltarono gli aspetti nazionali si moltiplicarono nell'ultima parte dell'Ottocento con la cosiddetta «monumentalizzazione delle città» e cioè la 
costruzione di monumenti celebrativi, uffici pubblici dalle grandiose dimensioni, la creazione di giardini ornamentali. Particolare rilievo ebbe la statuaria commemorativa riguardante personaggi di rilievo nella storia patria o comunque riconducibile a figure di spicco nella vita culturale nazionale, spesso le stesse esaltate in modo ripetitivo anche nei libri di testo (le tombe di Dante e di Garibaldi, il campanile di Giotto a Firenze, il foro romano, il monumento alla vittoria di Legnano, ecc.). Una seconda ondata monumentale coincise con la stagione fascista che ebbe grande impatto sul paesaggio urbano con imponenti costruzioni in stile modernista che ambivano a gareggiare (specie nella capitale) con la grandezza romana.

La presentazione della realtà rurale fu per molto tempo, almeno fino ai primi decenni del Novecento, condizionata da due stereotipi. Il primo era legato a una visione idilliaca della campagna e dei suoi abitanti, una sorta di «dolce arcadia nella quale i contadini sono lieti di svolgere i lavori dei campi». Il contado era descritto, per esempio, nei libri scolastici come un ambiente sano, ricco di valori tradizionali in cui la semplicità della vita si coniugava con la genuinità dei cibi, un luogo adatto a trascorrere il período di riposo estivo. Il secondo stereotipo era l'inferiorità manifesta delle abitudini di vita dei campagnoli rispetto alle abitudini "più civili» proprie delle comodità della città. Sarà il fascismo a imprimere una svolta a questa impostazione attraverso le campagne volte a valorizzare la vita rurale e a inserirle nel flusso della modernità, un impegno non disinteressato, ma perseguito con l'intento di legare al regime popolazioni molto attaccate alle tradizioni popolari e diffidenti, almeno inizialmente, al fascismo.

Nell'ultima parte del libro Targhetta presenta i paesaggi educativi della montagna, a lungo poco considerati nell'immaginario collettivo perché isolati, di accesso difficile, impervi e luogo di svago di piccole quote di appassionati alpinisti. Sarà la prima guerra mondiale a modificare questo stato di cose e a far conoscere a tutti gli italiani il paesaggio alpino con migliaia di giovani soldati arroccati sui confini alpestri per difendere la Patria e protagonisti di racconti epici veicolati dai giornalisti di guerra e dai primi cinegiornali. Le Alpi diventano così da «barriera naturale che protegge l'Italia dalle incursioni straniere a sacrario della Nazione, monti sacri in quanto bagnati dal sangue italiano». 
Questa presentazione del libro di Targhetta rende solo parzialmente l'ampiezza della ricerca e degli spunti di riflessione. Il volume è di grande efficacia anche per la capacità narrativa di Targhetta e dimostra come la ricerca educativa abbia grandi potenzialità interpretative quando sa situarsi all'intersezione di più competenze scientifiche ed avvalersene in modo critico.

Giorgio Chiosso

Università di Torino giorgio.chiosso@unito.it 